\title{
Study on Social Influence on Preschool Children by Sports Games
}

\author{
$\mathrm{Li} \mathrm{Li}$ \\ Shaanxi Xueqian Normal University \\ Xi'an, China
}

\begin{abstract}
As an ancient cultural phenomenon, games were generated since the emergence of human civilization, and have a significant impact on human's production and life activities. In the course of preschool children education, the study on social influence on children by sports games will be of positive significance for the formulation of scientific and reasonable education program for preschool children.
\end{abstract}

Keywords-traditional culture; middle school Chinese; classroom teaching

\section{INTRODUCTION}

With the emergence of human civilization, the human activities of games were given birth gradually. So it can be said games have been existing ever since the generation of human beings. Game is a relatively common social activity, which is an important part of social activities. Sports games are an integral part of games, so only obtain correct understanding of the meaning of games and sports games, can we accurately recognize the social impact of sports games on preschoolers.

\section{UNDERST ANDING OF SPORT S GAMES}

To understand relevant knowledge of games and sports games in-depth, we need to learn about their origin, characteristics and role to truly understand the significant social impact on preschool children by sports games.

\section{A. Origin of Games}

Game is an ancient and popular activity which appeared accompanying the emergence of human, and game activities are closely related to our daily lives. Usually, the orig in theory about games involves the following versions: 1 . Games are originated in primitive religious worship and witchcraft activity, which are to pray to the gods for blessings; 2. Games are produced to pass down the original culture; 3. Games are generated gradually in human labor process; 4. Games are excited by animal instinct which are born with the birth of species, and animals may adapt to environment better through games [1].

\section{B. Characteristics of Games}

From a functional point of view, games are uncertain with freedom; from the point of view of places where they occur, they tend to present dynamic characteristics, and the game courses are often repeated with sufficiency; from the perspective of the player's evaluation, games have the nature of fun and relaxing; from the perspective of active structure, game are loose without fixed form.

\section{Role of Sports Games for Children's Development}

First of all, physical health. The participation in the activities of sports games will be of positive effect to the growth and control of children's feeling and sensory system. In the process of sports games, children's movements have strong features of repetitive and sequential, enabling children to establish a systemic sensory and muscle system, thus to contribute to the health growth of children, as well as to promote the continuous development of their body movements' coordination and athletic ability.

Secondly, children's thinking. Children's participation in various sports and activities will be of important effect for their understanding ability. In the course of children's participation in activities of sports games, the promotion of games and enhance of students' understanding will influence and interact with each other. Through sports games, children may get more complex cognition, and give feedback to practice through games, so as to obtain more complex cognition. Such interaction can promote the development of children's senses, thinking ability and other aspects [2].

Again, emotion and mood. Children's emotion can be adjusted through games, so as to cultivate children to have a more positive and optimistic mood. Children's negative emotions can be transferred through sports games, and their anxiety and feeling of fear generated in social life can be erased through positive guidance. This also allows children to have a better understanding about themselves and to get pleasure.

Finally, children's social roles. By participating in sport games, children may have a more detailed understanding of their social roles. In the course of playing games, children can gradually learn about relevant social culture and social customs and certain rules of communication, and can establish good relationship with others who participate in the same game, so that children may generate some sense of belonging to society to promote the development of their social behavior [3].

In addition, games also have a positive effect on children's language development. The process of sports 
games provides children certain occasion and environment to learn language skills. In the course of playing games, children may share their own thoughts, emotions and other aspects with their partner and parents. And they are able to describe their behavior using language, which may help children to apply more clear and coherent language to express and convey information, so as to promote continuous enhancement of children's interpers onal skills.

\section{SPORT S GAMES AND SOCIABILITY OF PRESCHOOL CHILDREN}

Through sports games, children can get a lot of knowledge and awareness, which have significant impact on parent-child interaction, peer interaction and social behavior of preschool children.

\section{A. Social Development of Children}

The so-called socialization of children refers to that children are able to interact with social environ ment, so as to obtain various behavioral norms, value ideas, knowledge and skills of the society they live in under the influence of social environment. Generally, it will be expressed as three specific behaviors as parent-child interaction, peer interaction, social behavior. In the process of children's gradual socialization, namely the process of children to gradually adapt to social life as an independent member of society, children are usually required to be offered with basic life skills education, to promote the development of self-concept, develop good habits, develop good moral character and foster social roles. In the socialization process, children can form their social development and personality through continuous practice and cognition, thus to make children come to realize their role and status in society, to have a more objective mentality, to be able to control their behaviors, and to meet social norms.

\section{B. Games and Parent-Child Interaction of Pre-school Children}

Psychologists Freud believes that childhood games can help children release their pressure generated by repression and constraints of social rules, so as to eliminate their tension and avoid unpleasant phenomenon or event.

During parent-child interaction, parents will adopt the behaviors of power control, removal of care and guidance to influence and guide children's behavior, namely behavioral reinforcement. Behavior reinforcement refers to that in the process of parent-child interaction, children's behavior will be changed by their parents according to their different reactions. If a child to makes a mistake or do not listen to their parents, the parents usually have him/her to be punished through power control, thus to encourage children to actively change their behaviors in violation of rules. Due to the limitation of children's parents and external social environment to improve their life, children cannot to satisfy their wishes in accordance with their own ideas. Such constraints and pressure from outside world will make children generate anxiety, resulting in their incompetence with different degrees in terms of emotions and behavior [4].
At this time, we need to make help children to escape coercion and restraint of real world through sports games, show their bitter experience, and gradually to change their role and to control positively. Through sports games, children may gain a sense of control, and make judgment about the environment and the effectiveness of environmental behavior in the process of playing games, to see what kind of behavior is allowed. In the process of recognition of their behavior, children will gradually recognize the positive emotional experience.

In children's early stages of growth, they get experience in the process of contact with their parents, thus a good parent-child relationship is of an important role to the healthy growth of children. Therefore, through the establishment of harmonious parent-child sports games, their accumulation of experience can be effectively promoted. In the parent-child activities of sports games, parents can not only teach some life skills to children through the games, but also can guide children to express their thoughts through games by setting themselves as models. Through parentchild sports games, children can not only get their parents' attention and care, but also can obtain pleasant mood in the process of strengthening expression, thus parent-child relationship is strengthened. By building a harmonious parent-child interaction, children's mental health can be guaranteed, and their early experience can be enriched, helping children to develop a healthy personality, thus to promote their social development.

\section{Games and Peer Interaction of Preschool Children}

Sports game is an important form for children to interact with companions, which also provides necessary conditions for children to make exchanges and communicate with their companions. Peer interaction activities must be carried out on the basis of children's positive initiating and participation in the activities, requiring children to have the judgment and coordination ability about others attitudes and communication intention. Children are also required to play a certain role in course of communication in order to ensure a smooth peer interaction [5].

First of all, children have high selectivity when selecting the roles they will play and imitate, and they will also make adjustments according to different social expectations. Adjustments shall be made timely according to children's inherent emotional needs. In the process of interaction with peers, children tend to play a leader's image and the one who is inclusive when they feel they are being needed.

Secondly, while children are involved in sports activities, they are not simply involved in the game, but also play games with profound emotional factors. In the process of role selection, children often choose the model toward whom they have a feeling of love, respect, jealousy or anger, by imitation those models, especially their favorite roles, they will generally realize the expectation of "the same as adults". In common sports games, children will usually choose to play the king, queen, fairy, superman and other roles, which show their strong psychological needs. In addition, children will also imitate some people or things that make others fear, 
thus to conquer the sense of fear. Through playing the image with conscious, children's centralized process development will be promoted, and they can make self-examination from other point of view, so as to promote the development of cognitive skills and self-awareness [6].

Finally, through interaction with companions in sports games, children can gradually get through the stage of mother fixation and gain independence. Children will be restricted by rules while participating in sports games, so as to nurture their capacity of self-control. In the process of playing sports games, children will formulate game rules as the game progresses. On the one hand, it can make children understand the rules in-depth and clarify the nature and characteristics of rule-making, helping them to learn certain social rules while communicating with companions, so as to promote social behavior to continuously develop toward the friend and standardized direction.

\section{GAMES AND PRESCHOOL CHILDREN'S SOCIAL BEHAVIOR}

The so-called social behavior refers to people's attitudes and reactions reflected through someone or some thing in the process of social interaction. According to different expressions of individuals in social behavior, namely the motivation and purpose of social behavior, generally social behavior can be divided into: pro-social behavior and antisocial behavior. One reasonable manner and approach may clear up the aggressive anti-social behavior effectively, and sports games can effectively reduce anxiety and release their aggressive behavior. In children's growth process, es pecially when they encounter difficulties, they will feel anxious even with a sense of grievance, and if they cannot control their emotions, games are needed to release those feelings. Generally, the typical ones are angry talking, loud yelling, frantically running and jumping in process of playing sports games, and transfer their emotions to toys, such as beat, damage and throw about the toys. Those behaviors will be stopped when the children have let off their discontent, and gradually restore calm [7].

Therefore, sports games can be side being a way of selfexpression for children, which can also provide emotional outlet for children, enabling children to gradually learn to control their emotions and learn how to live in harmony with others in their growing process. While playing sports games, children gradually learn to apply their own approach to solve problems encountered in society. Teachers and parents shall pay attention to children's emotional changes in teaching and getting along with them, and guide children to participate in sports games, so as to promote the cultivation and development of their sociability.

\section{CONCLUSION}

In summary, thanks to their own characteristics, sports games are the main activities for preschool children with great significance to promote children's physical and mental development. Sports games are action-oriented which are easily to be accepted by children. Through various activities and movements of sports games, children can acquire new knowledge and awareness and improve their life skills effectively. And they will come to realize social norms gradually while playing games, enabling children to grow up in a relatively relaxed and pleasant environment to acquire new knowledge. Therefore, in modern preschool children education, parents can take reasonable advantage of sports games to strengthen interaction and exchanges with children, enabling children to form a healthy personality gradually while they are growing up.

\section{REFERENCES}

[1] Kuang Quan, Xu Dong. Social Development of Games and Preschool Children---Game Theory based on Psychoanalytic School [J]. Journal of Taiyuan Normal University (Social Sciences), 2015(1)

[2] Wang Minghui. Analysis of Influence on Cognitive Development of Preschool Children by Sports Games [J]. Intelligence, 2013(33)

[3] Guo Jingshuang, Pan Xiuli, Wang Zuopeng. Influence on Psychological Development of Preschool Children Aged 3-6 by Sports Games [J]. Journal of Harbin Institute of Physical Education, 2012(2)

[4] Yan Hui. Influence on Social Development of Children by Games [J]. Business Culture (First half of the month). 2011(09)

[5] Feng Jingdan, Li Sixian. Study on Influence on Social Development of Children by Folk Games [J]. The Modern Education Journal, 2015(1)

[6] Xu Yifen. Promotion of Preschool Children Socialization by Games [J]. Journal of Ningde Teachers'College (Philosophy and Social Sciences Edition), 2015(1)

[7] Guan Bo, Yang Zhaoshan, Zhang Mo. Study on Social Development of Children Aged 5-6 by Scene Sports Games [J]. Journal of Shenyang sport University, 2013(1) 